\title{
Duality in topological algebra: Addendum
}

\section{B.J. Day}

It has been observed by Banaschewski [1], Proposition 1, that, in the notation of the author's paper [2], $\bar{P}=P^{\prime}=$ Pro $P$ if $P \subseteq F U$ is hereditary and finitely productive. This fact does not require the use of injectives as in [2].

Thus, under the preceding hypotheses on $P$, we have the following:

PROPOSITION 1. The inclusion P P ProP is codense (that is, $A \cong \int_{P}\{u(A, P), P\}$ for all $A \in$ Prop $\}$.

Proof. We have $P \subset P r o P \subset U$. Let $E$ denote the subcategory of $U$ whose objects are those of $U$ and whose morphisms are the regular epimorphisms (equals coequalisers) in $U$. Let $H=E \cap P$. Then, because $A \in$ Prop , we have $A \cong \int_{P \in H}\{E(A, P), P\}$. The canonical map $\int^{Q \in H} E(A, Q) \times u(Q, P) \rightarrow U(A, P)$ is an epimorphism for all $A \in$ ProP and $P \in P$, since each map $f: A \rightarrow P$ factors as $A \rightarrow Q>\rightarrow P, Q \in P$, as $P$ is hereditary. Thus there is a monomorphism

$$
\begin{aligned}
\int_{P}\{u(A, P), P\} & >\int_{P}\left\{\int^{Q \in H} E(A, Q) \times U(Q, P), P\right\} \\
& \cong \cong \int_{Q \in H}\left\{E(A, Q), \int_{P}\{U(Q, P), P\}\right\} \\
& \cong \int_{Q \in H}\{E(A, Q), Q\} \text { by the representation theorem, } \\
& \cong \because .
\end{aligned}
$$

Received 13 June 1978. 
By considering the appropriate diagram (see [3], Theorem 2.3) one has that this monomorphism is left inverse to the canonical morphism $A \rightarrow \int_{P}\{U(A, P), P\} ;$ hence is an isomorphism. //

PROPOSITION 2. There is a duality between ProP and the $G$-copresentable algebras from $P$ to Ens where $G: P \rightarrow$ Ens is the forgetful finctor.

\section{References}

[1] B. Banaschewski, "On profinite universal algebras", General topology and its relations to modern analysis and algebra III, 51-62 (Proc. 3rd Prague Topological Symposium, 1971. Academia Publishing House of the Czechoslovak Academy of Sciences, Prague; Academic Press, New York, London; 1972).

[2] B.J. Day, "Duality in topological algebra", Bulz. Austral. Math. Soc. 18 (1978), 475-480.

[3] B.J. Day, "On Pontryagin duality", Glasgow Math. J. (to appear).

Department of Pure Mathematics, University'of Sydney, Sydney, New South Wales. 